\title{
LA GESTIÓN DE LA INVESTIGACIÓN: DIFERENCIACIONES Y RELACIONES
}

\section{The management of research: differentiation and relationships}

\author{
William Mantilla Cárdenas' \\ Recibido: 3 de agosto de 2007 • Revisado: 25 de septiembre de 2007 - Aceptado: 12 de octubre de 2007
}

\begin{abstract}
Resumen
Este artículo forma parte de la línea de investigación en el campo disciplinar de gestión de la investigación. Específicamente mediante un análisis de contenido e investigación documental, se explica y se argumenta con base en la recolección de datos, por qué referirse a la gestión de la investigación como campo central de estudio e investigación diferenciable de campos relacionados, tales como "Gestión de ciencia tecnología” y "Gestión del conocimiento". Todo ello mediado por la superación de equívocos y reduccionismos. Si es necesario diferenciar, de una parte también es preciso establecer las relaciones, que se considera como algo más importante en la práctica. Mientras que la diferenciación tiene más importancia desde el punto de vista académico, sobre todo por que esta investigación forma parte de los resultados que llevan a un Programa de Maestría propuesto.
\end{abstract}

\section{Palabras clave}

Investigación, gestión de la investigación, ciencia, tecnología, epistemología, ciencias administrativas.

\begin{abstract}
This article is part of the investigation line in the dicipline field of management of investigation. Specifically by means of a content analysis and documental investigation, it is explained and argued in terms of data collection. Why to refer to management of investigation as central field of study and investigation, diferent of related fields, such as "Management of science-technology" and "Management of knowledge." All that mediated by the mastering of misunderstandings and minimaxings. If it is necessary to differ, from one part it is also necessary to
\end{abstract}

Docente investigador Unidad de Investigación y Posgrados USTA. 
stablish the relationships that are considered as something more important in practice. While the differentiation has more importance from the academic point of view, mainly because this investigation is part of the results that are taking to a proposed Master Program.

\section{Key words}

Investigation, management of investigation, science, technology, epistemology, administrative sciences.

\section{Excurso sobre los reduccionismos}

A continuación se plantean los equívocos principales y los reduccionismos más frecuentes que se originan de este concepto, lo cual permite mostrar la importancia e ir aclarando a lo que se pretende referir con la gestión de la investigación, antes de pasar a la exposición de las relaciones y diferencias.

En principio existe una marcada tendencia a vincular la gestión de la investigación con la gestión de recursos en los centros de investigación. Este concepto aunque muy popular por que es la primera intuición de quien se aproxima a este conocimiento, es equivocada y reduccionista. Se debe pasar a explicar las razones por la cuales esta definición se considera equivocada, y los diferentes reduccionismos que deben ser superados para fortalecer, no sólo un enfoque o una defensa teórica sino que en la práctica se pueden encontrar alternativas creativas y creadoras de conocimiento, pertinentes y adecuadas que por rigurosas no dejen de ser audaces en la búsqueda de conocimiento que más allá de buscar soluciones a problemas nos haga una sociedad creativa, sabia y feliz.

El concepto señalado es equivocado desde la estructura de pensamiento misma, de la cual proceden las definiciones como de glosarios y de diccionarios. Se pretende entonces simplificar la gestión de la investigación a una definición que no explica la totalidad del campo como un campo de conocimientos implícitos y explícitos; $y$ de prácticas adecuadas $o$ inadecuadas; eficaces o no.
En este caso la tendencia general es a definir la gestión de la investigación como un servicio. Se toma como ejemplo la siguiente definición sobre la cual se puede señalar su coincidencia con la definición planteada al iniciar el artículo desde esta perspectiva: Para comprender la actual situación de los servicios de gestión de investigación de las universidades es preciso recordar que su aparición es relativamente reciente. [En España] Es a partir de la Ley de la Ciencia, en 1986 cuando las universidades empiezan a crear estructuras dedicadas a la gestión de la investigación, con distintas funciones y con distintas dependencias orgánicas y funcionales. La definición y el pensamiento que ubica la solución de la producción de conocimiento por medio de la formalidad y la creación legal versus la creación cognitiva con respecto a las universidades y la creación de centros de investigación por decreto no se aleja mucho de la realidad de la producción de conocimiento en los países latinoamericanos y particularmente en Colombia.

Pero lo importante de señalar en el contexto es la relación entre definición y servicio para dar cuenta de la gestión de la investigación y entonces dejar por fuera la incidencia de la gestión en el mismo curso cognitivo de las prácticas investigadoras, ya sea situadas en el contexto de proyectos de investigación como es la forma generalizada y ligadas a macro procesos investigativos, tales como: campos o líneas de conocimiento que permiten organizar la compleja acción investigativa. 
En el contexto reciente de la Educación Superior en Colombia la investigación se ha impulsado a partir de una presión, de organismos acreditadores más que por una real convicción generalizada en el sector y surge entonces como una consecuencia la necesidad de gestionar investigación, lo que de paso muestra que la posibilidad de gestionar conocimiento no está al alcance.

Este crecimiento heterónomo de la investigación hace que tenga consecuencias en la gestión, dado que afecta la pertinencia y significatividad. La cuestión puede quedar reducida a reportar cierto número de grupos de investigación y ha dedicar tiempo de investigación lo cual es positivo, pero menos contundente que una perspectiva más autónoma de desarrollo y consecuente con uno de los principios más recurrentes por parte de las universidades que se denomina justamente a "Autonomía Universitaria" consagrada en la Ley correspondiente.

La gestión de conocimiento incluye conocer las empresas que producen algo que otros necesitan. De hecho, la página del Stocker Group ${ }^{2}$ genera en Latinoamérica un intercambio de negocios entre la pequeña y la gran empresa. Desde la perspectiva de la gestión del conocimiento lo que se trata de solucionar es la falta de conexión de productos y de servicios. Este modelo de negocio para generar confianza en la realización virtual de negocios ha generado en 2 años, 60.000 conexiones de negocios por 200 millones de dólares en 9 países de América Latina que incluyen a Colombia. Estas ruedas de negocios son una de las prácticas más difuminadas para la gestión del conocimiento.

En su versión original, la gestión de conocimiento se refiere a la gestión de resultados de conocimiento con base en un valor agregado generado por las organizaciones empresariales. Pero desde esta perspectiva en el contexto de la educación superior que en nuestro país promueve la mayor cantidad de producción de conocimiento la pregunta es: ¿Cuál conocimiento para gestionar? O existe, pero no es visible o no hay producción de conocimiento que permita justificar resultados de conocimiento para gestionar.

La gestión del conocimiento no siempre está ligada a la producción del conocimiento, en algunos casos se define dentro de la misma perspectiva de servicios que se ha señalado para la gestión de la investigación así: "conjunto de procesos y sistemas que permiten que el Capital Intelectual de una organización aumente de forma significativa, mediante la gestión de sus capacidades de resolución de problemas de forma eficiente, con el objetivo final de generar ventajas competitivas sostenibles en el tiempo" (Maroto, 2007). De hecho esta definición tiene como referente a la organización aislada y el conocimiento como lo que se produce en el entorno interno de la organización.

Pero bien es preciso señalar que la gestión del conocimiento en un enfoque más evolucionado $y$ sinérgico se refiere a la minería de daos y a la memoria corporativa. Que son las formas de generación de conocimiento significativo para el desarrollo y el aumento de la productividad organizacional. Se trata entonces de hacer visibles producciones cognitivas empresariales, y hacer conciencia de los logros que esta inventiva contextual produce en los resultados de la organización y específicamente del negocio.

Desde este punto el enfoque principal de la gestión del conocimiento se encuentra en el medio empresarial y esto es muy significativo, dado que entonces cumple un papel especialmente ligado a la productividad y el aumento de mejores oportunidades de negocios. Tanto en la capacidad de la empresa para lograr sus objetivos (conocimiento estratégico) como para solucionar problemas internos de producción y de procesos en los servicios empresariales (conocimiento situacional). En cualquiera de los dos niveles señalados y de los dos tipos de gestión de conocimiento, se trata de la conversión del conocimiento en capital.

www.stockergroup.com. 
Sin embargo, ahora es más claro que la gestión de conocimiento debe estar ligada al proceso de producción de dicho conocimiento, sea que proceda de inventiva organizacional o de procesos de producción más ligados a procesos de investigación.

Pero es necesario anteponer que el discurso empresarial de la gestión de conocimiento se caracteriza, desde este punto de vista, por su despreocupación por la naturaleza de dicho conocimiento, o por su proceso de construcción y de producción; lo cual hace que se diferencie mucho del discurso de gestión de conocimiento que circula en los medios universitarios o de educación superior que se encuentra instaurado de manera dependiente de una organización que distribuye el conocimiento por disciplinas y que tiene una alta supeditación del mismo a la epistemología y a la lógica desde la cual procede y se produce dicho conocimiento integrado a la investigación y a las Ciencias. Esto llevaría al análisis de las ciencias administrativas en el contexto académico y su relación con las prácticas empresariales, en el marco de la gestión del conocimiento y de la investigación que excede las posibilidades de este artículo.

Ahora bien, que el papel de la gestión del conocimiento como servicio y como demanda también decide el tipo de conocimiento que es necesario producir. Si esto es así, como se muestra en el siguiente texto representativo, la discusión o más bien los alcances van más allá de un mero servicio o de una definición instrumental de la gestión de la investigación, pues toca de manera directa la validez y la justificabilidad de las investigaciones en áreas propiamente epistemológicas de la producción investigativa en particular, y de la producción de conocimiento en general.

Lo anterior es útil también para mostrar que la gestión del conocimiento pertenece a un contexto diferenciable de la gestión de la investigación. La complejidad del punto obliga a especificar el uso, no ingenuo, de la expresión diferenciable frente a la taxativa de: "diferente". Diferenciable significa que se puede hacer diferente y no que necesariamente lo sea. Es decir, que pueden estar relacionados tanto en contextos: el de la Educación Superior sobre el cual se hace especial énfasis y las universidades específicamente; como en propósitos: vinculados a los centros de gestión de conocimiento españoles estudiados para esta investigación como ejemplo de prácticas de gestión de la investigación. Allí también se considera a la gestión de la investigación como un servicio, una actitud muy cercana a los inicios, hace apenas una década de la gestión del conocimiento y que se ha criticado desde la reducción a la definición.

El entrecruzamiento de los contextos y la hipervinculación de los nexos como características preponderantes del curso actual, hace sospechar que un discurso analítico y taxativamente diferenciador, se encuentra en contravía de la interpretación actual de las teorías actuales de la gestión de conocimiento, y más aún, de la gestión de la investigación. El título de un libro muy utilizado en cursos de formación muestra esta circularidad de productor - producido a la que se refiere Morin en los principios de la complejidad: "La Gestión del Conocimiento y la utilización de las tecnologías de la Información y de las comunicaciones en la creación de valor en los proyectos de Innovación"

Es entonces mayor el énfasis que se quiera poner en el conocimiento aplicable a la solución de problemas sociales y tecnológicos. Es decir conocimiento útil y de manera inmediata, pero ¿Puede considerarse la investigación desde este propósito de manera explícita e insistente y puede verificarse de antemano en la producción de proyectos la relación de resultados que se evalúan a priori con indicadores del éxito en este sentido? Es una pregunta a la vez de gestión y es en sí misma una pregunta de conocimiento. Es decir, una pregunta sobre la gestión de conocimiento, ya no sólo en la innovación sino en la investigación y definitivamente entramos en otro terreno.

Se pasa por alto este punto en la gestión de la investigación como servicio y como definición, especialmente desde la definición tipo citada al inicio de este artículo. Veamos: “El papel de las universidades y muy 
específicamente el sistema de educación superior, tiene sin duda una responsabilidad ante la sociedad en su conjunto, ya que esta última exige a la universidad producir, entre otras cosas, conocimiento científico socialmente válido capaz de generar soluciones creativas en las múltiples áreas del quehacer social. Estas exigencias según, Tûnnermann se perfilan por las siguientes razones socio políticas:

- La pérdida de calidad y efectividad social de estas instituciones que ponen en alerta al ente financista de las misma, el Estado, que a su vez es responsable de dicha crisis.

- La acentuada crisis de los sistemas de planificación educativa como acción correctiva de los sistemas institucionales.

- La dualidad y contradicción de la organización del sistema de educación universitario.

- La herencia política indiferente y cómplice a la crisis del sector.

- La asignación presupuestaria irrisoria.

- La intención de control total por parte del Estado obstaculizadora de la necesaria autonomía.

- La creciente demanda de los demás sistemas sociales a la educación como símbolo de desarrollo.

- La ausencia de una cultura de la planificación y dirección sistemática de estas organizaciones.

- La utopía de la masificación versus la calidad

- La necesidad de mejorar los sistemas institucionales.
Sobre la base de estas exigencias,"las funciones de la universidad adquieren también una nueva dimensión frente a la revolución socio-científica, provocada por el papel central de la ciencia en la sociedad post-industrial" (Tûnnermann, 2002, p. 96).

La relación producción de conocimiento, gestión de la investigación y desarrollo nacional es más clara, pero también más mecánica. En primer lugar no es posible referirse a un sistema organizacional como referente y en segundo lugar es imposible desligar el conocimiento de sus formas científicas de producción:

La realidad latinoamericana sobre los resultados obtenidos en los sistemas de investigación universitarios, tiene sin duda una noción muy particular en lo referido a la producción, apropiación y aplicación de conocimientos y tecnologías. Cada país del continente tiene su propia visión sobre la gestión de la investigación y los resultados alcanzados por los esfuerzos desarrollados por estos países se desconocen a grandes rasgos Royero (2002).

Lo que Guibons ${ }^{3}$ refiere sobre los modos de producción de conocimiento entra en juego en relación con la gestión de la investigación y sus posibilidades como alternativa generadora de desarrollo. Las diferencias que refiere Royero sobre las particularidades en la visión que cada país tiene de su gestión de la investigación no otra cosa que la falta de estudio de gestión como un campo cognitivo. Es de hecho una perogrullada plantear que un proyecto de investigación es también, tanto en su diseño como en su desarrollo, sistematización y hasta su publicación y apropiación por la comunidad científica; un proceso de gestión sin el cual todo el esfuerzo intelectual es vano, pero también es vano plantar que todo ello va directo al desarrollo nacional.

Ver en Guibons (2003) modo 1 y modo 2 de producción de conocimiento. Más adelante se hace una referencia a estas definiciones claves para validar y resignificar conocimiento en países latinoamericanos y para hacer visible conocimiento existente no formalizado pero diferente del conocimiento implícito como concepto de la gestión del conocimiento. 
La versión mecánica no corresponde a Guibons ni de Royero; pero sí a la burocracia técnica que plantea la ecuación más o menos establecida en políticas nacionales de investigación que se puede enunciar como: Educación + Investigación = crecimiento y por carambola a desarrollo. Ya Habermas lo planteaba en mayores proporciones con su referencia al positivismo ingenuo que piensa que las ciencias están directamente relacionadas con el bienestar y que se puede ver en el mundo de la vida de una manera equitativa el desarrollo científico técnico. Las fuerzas del mercado y la política se hayan pendientes de controlar y afectar la producción científico tecnológica, tanto en su momento creativo como en su conveniencia de resultados: esta es una de las discusiones más importantes sobre responsabilidad social y sus variables ecológica y social.

En el discurso burocrático sobre la educación superior en general y sobre las universidades en particular recae una de las posibilidades y alternativas de generar desarrollo en sentido amplio, a partir de una gestión de conocimiento y más de una gestión de la investigación, al menos en el caso colombiano. Todavía más reducido la concepción.

Es entonces clave comprender en primer lugar, que tratar de acuñar el concepto tanto de gestión del conocimiento como de gestión de la investigación en una definición o sea en una fórmula resumible es equivocado, por que la gestión de la investigación más que un concepto que se defina, constituye un conjunto de interdisciplinario de saberes que tienen diversas perspectivas y enfoques. La gestión de la investigación no se reduce a la gestión de proyectos de investigación, ni a contextos organizacionales tales como la gestión de centros de investigación o la investigación en IES. Tampoco es reducible solo a un servicio y de otra parte tampoco es independiente de la episteme de cada acción científica o de cada proyecto de investigación como unidad básica de la gestión de conocimiento.

Por ejemplo es también clave comprender que la ad- ministración y la gestión de un proyecto experimental de investigación es muy distinta a la gestión de un proyecto de corte etnográfico; y esto sin cruzar aun las diferencias disciplinares y la necesidad de proyectos inter y trans disciplinares.

De otra parte como ya se ha mencionado, es clave establecer las contradicciones cualitativas y cuantitativas de diferenciación de niveles de gestión de la investigación según su referente y no sólo su contexto. Es válido referirse a un nivel organizacional en la primera versión de la gestión de conocimiento; pero no es válido referirse a un nivel organizacional en la gestión de la investigación. Allí lo que procede es referirse a un nivel de redes de investigadores o al contexto de aquel o de aquella organización, centro $o$ incluso unidad organizacional.

Pero lo más significativo para la gestión de la investigación en su nivel de aproximación más básico es su relación con el ciclo PHVA de las ciencias de la administración que procede de la gestión integral y también de la consideración de proceso de los sistemas de gestión de calidad. Como se puede ver más adelante esto refiere al nivel más básico por que en otros niveles es necesario remontarse desde las disciplinas de las ciencias administrativas a la sociología del conocimiento y a la epistemología para comprender la gestión de la investigación como un proyecto de conocimiento.

También en el nivel básico es necesario mostrar que además de centros o unidades organizacionales se gestionan sistemas de investigación, redes; desde nacionales hasta internacionales vinculadas a mega proyectos investigativos o a colaboraciones en líneas de investigación. La gestión del conocimiento tiene la doble adscripción no necesariamente consecuente como suele suceder con la mayoría de las disciplinas, de elementos para la comprensión y de elementos para la praxis dentro de los cuales se encuentran los elementos de gestión.

Lo planteado es referido a los elementos para la 
comprensión. En cualquier contexto práxico la gestión disciplinar es una coordenada que remite a las reglas básicas de producción cognitiva de esa disciplina. La variable disciplinar afecta de manera importante la gestión de la investigación y viceversa, incluso la forma como se reportan los resultados.

No sabemos cómo esto ha sido reportado por la filosofía de la ciencia. Por ejemplo, no sabemos la relación que esto pueda tener con las preocupaciones de Kuhn sobre el cambio científico. Esto obliga a una relectura del autor desde una perspectiva de la gestión del conocimiento. No sólo como se hace actualmente al referirlo a manera de paseo intelectual. Esto vincula la epistemología, la política, la ética y la incidencia de las disciplinas de la administración como se muestra más adelante.

Un punto determinante de la gestión de la investigación, dejando atrás los contrastes con la gestión del conocimiento y que ha permitido su emergencia disciplinar, consiste en aclarar que lo que se gestiona es la acción investigativa, pues la investigación tiene su propia lógica y su episteme vinculada al problema de conocimiento que se pretende resolver y a la justificación racional del proceso investigativo no sólo a su utilidad. El proceso investigativo más básico puede generar el conocimiento más útil aunque no sea su finalidad, ya sea por el efecto carambola "serendipity" o por su capacidad para generar procesos comprensivos. Es muy frecuente que las ciencias sociales usen teorías y categorías de las ciencias naturales de una forma metafórica y que acertada o equivocadamente resulten explicando o interpretando fenómenos sociales.

Pero justamente el desconocimiento de las lógicas y de los procesos puede llevar al fracaso o a problemas éticos de la investigación. La investigación en consecuencia, requiere de proceso gerencial muy diferente $y$ de una planeación muy específica que a su vez pueda constituir indicadores de evaluación más claros. He aquí la primera forma en la cual aparece la gestión desde una perspectiva integral como un proceso de planeación, gerencia y evaluación de la calidad de los procesos y de la acción investigativa.

Lo anterior lleva a un problema de orden epistémico y no sólo de gestión lo cual hace que emerja un campo disciplinar. Entonces es un problema de orden epistémico sobre la incidencia de la administración, de la gerencia o de la gestión investigativa en general, en la producción misma de conocimiento. He ahí la necesidad de hablar de referirnos a una disciplina emergente y la forma como el primer reduccionismo de la definición queda en evidencia.

\section{Nivel básico de la gestión de la investigación en relación con las disciplinas administrativas}

En relación con este primer problema de conocimiento, la alternativa es explorar los elementos de las disciplinas de la administración que permitan interpretar la forma como los investigadores, grupos, organismos o sistemas realizan la investigación como un proceso. En principio entonces interesa sobre manera analizar las formas como se planea, se gerencia, se evalúa y se mejora el proceso de investigación. El objeto cognitivo central y característico de las disciplinas de la administración se puede desprender del ciclo: planear, hacer verificar y actuar (PHVA) de Deming que anteriormente fuera señalado por Shewhart ${ }^{4}$ desde los años treinta del siglo XX.

Este ciclo se concibe como un holograma estructural para la concepción de la gestión de la investigación, en la cual el planear corresponde con la hipótesis de la planeación estratégica interactiva. El hacer corresponde con el sentido de la gerencia pluri personal, el verificar corresponde con el sentido básico de evaluar la calidad de los conocimientos y del proceso desa-

Ver: Deming Calidad, Productividad y copetitividad: la salida de la crisis. Madrid: diaz santos, 1989 y Shewhart Economic Control of Quality Of manufactured Product. Mac Millan, Nueva York: Reeditado por la American Society For Quality control, 1980. 
rrollado para lograrlo y el actuar con la concepción de mejoramiento o hacer cualificado por el aprendizaje de la acción ${ }^{5}$.

La planeación estratégica incorpora la necesidad medios fines de mediano y largo plazo que en la investigación se complejizan de una manera importante, pues la investigación y la gestión constituyen un trabajo de producción cognitiva ligado a líneas de investigación o a campos de conocimiento y según la teoría que se reconozca más pluasible de manera provisional, a los paradigmas de Kuhn o los programas de Lakatos, muy diferentes y no exclusivos. En cualquiera de los casos es hablar de períodos históricos considerables.

Es interactiva dado que tiene un sentido heurístico, prospectivo y hermenéutico. $O$ sea que basada en el lenguaje no puede ser construida por directivos sino que incorpora los investigadores como comunidad pues ellos son intelectuales de su quehacer y no sólo "operarios investigativos". Las formas excluyentes de planeación se encuentran en desuso pues no generan ni pertenencia, ni valor agregado y quedan en enunciados muertos. De hecho las teorías organizacionales sobre organizaciones que aprenden y organizaciones socialmente inteligentes han incidido de manera positiva en las prácticas de planeación estratégica.

La gerencia se denomina pluri personal pues está basada en equipos. De hecho la forma como se desarrolla la acción investigativa es en equipos humanos. De esta manera los equipos de investigación realizan muchas de las actividades propias de un gerente: toman decisiones, administran reuniones, organizan y gestionan tiempos, delegan tareas, entre otras, pero de una manera muy particular que no se puede explicar aquí, y que tiene su característica central en la concepción de comunidad de investigadores. Si existe un gerente o sea una gerencia unipersonal que pretende ordenar sobre la lógica de los resultados de la investigación como en cualquier otra empresa que busca de manera razonable el éxito, los proceso investigativos grave riesgo o la organización queda reducida se auto liquida por su propia paradoja.

La gerencia es en consecuencia un proceso y no una persona. Existe entonces en ese contexto un equipo que dirige unos equipos y la cuestión no es de autoridad sino de visión organizacional.

Como se puede deducir para resumir se está dando un salto del ciclo PHVA a procesos de gestión y de administración. Así la planeación estratégica interactiva (prospectiva y hermenéutica) se sitúa cognitivamente en el primer componente del ciclo, la gerencia pluri personal en el segundo y así sucesivamente.

\section{Advertencia sobre los mecanicismos: "prevenir mejor que curar"}

Es necesario tomar ciertas precauciones teóricas y lógicas frente a esta relación entre investigación y gestión que debe dar por resultado una gestión de la investigación como categoría única básica en relación con las disciplinas de la administración. Las proporciones son diferentes, los propósitos teóricos y los puntos claves de referencia también, entorno a la investigación y la gestión.

Hasta ahora el ciclo gira entorno a una referencia ambigua que puede situarse tanto en la organización como en el individuo. La alternativa de solución a este vacío es la incorporación del eje central en el cual el ciclo define la acción individual. Es decir actuar en términos organizacionales es realizar el ciclo PHVA. Pero esto debe ser consecuente tanto en la acción individual como en la interacción colectiva.

Es decir, también en los procesos de articulación y comprensión organizacional. Así en los niveles de

Para una profundización en los conceptos de planeación estratégica interactiva; gerencia pluripersonal y mejoramiento continuo como aprendizaje colectivo ver: Mantilla William. Gerencia planeación y evaluación en organizaciones educativas. Informe Colciencias, 1999. 
organización y sistema la gestión integral es la forma como se desarrolla el ciclo de planeación que es extraordinario, estructural; la gerencia que es el día a día de la organización en contraste con el anterior es el quehacer cotidiano de la organización y del sistema; la evaluación y el mejoramiento que son aplicados según su referencia. Nótese que al interior cada componente tiene su propio ciclo PHVA lo cual hace que este sea hologramático de manera envolvente.

La investigación en este sentido gira entorno a las concepciones de las disciplinas de la administración desde el punto de vista de la planeación la gerencia y la evaluación con su referencia a la calidad, que interpretan de mejor manera las acciones en el contexto de la investigación, ya sea con referencia a sujetos, grupos (equipos), centros u organismos y sistemas de investigación. Sus coordenadas organizacionales, la forma como actúan la incidencia de las disciplinas de conocimiento y de los objetivos de los proyectos y planes de investigación y el objeto sobre el cual desarrollan su acción en un contexto diferente si es universitario o de empresa privada.

Significa además comprender la imposibilidad e inadecuación del traslado mecanicista de modelos de la administración a la gestión de la investigación e inclusive a las organizaciones educativas. El traslado acrítico de modelos de la administración a la educación ha tenido efectos contraproducentes. No hay estudios que permitan establecer la incidencia de modelos administrativos en la investigación. Pero en educación por ejemplo, la aplicación del modelo de mercado, el de calidad total de manera generalizada en el contexto latinoamericano. Se trata de aprender de las disciplinas de la administración para la educación, pero no de imponer modelos. Esto refleja entonces una de las manifestaciones del reduccionismo sobre la gestión de la investigación.
Otro reflejo de este mismo reduccionismo lo constituye la discusión actual sobre la ética de la investigación. No se trata simplemente de seguir unas normas de buena conducta o de comprender las restricciones necesarias para los experimentos en humanos o en animales. Se trata de la responsabilidad por los resultados y los procesos investigativos.

Como se ha mencionado, otro problema es de orden ético que implica las libertades en la investigación y la administración de programas de investigación con fines prácticos o práxicos que se han venido discutiendo en relación con la ética de la investigación más allá del cumplimiento de ciertas normas o criterios epistemológicos. Una de las conclusiones a la cual llegan los autores de la ética de la investigación es que la ciencia como institución social debe confrontarse más allá de sus comunidades científicas. También se hace necesario distinguir el error como parte de la práctica científica del fraude, ya sea en los procesos como en los resultados ${ }^{6}$. Ahora que la pregunta sobre una ética global en un mundo con mercados globales es aplicable a la ciencia y su relativismo actual.

Los fraudes van, desde el falso cráneo de Pitdown descubierto en 1912 hasta que un odontólogo demostró que era un fraude; hasta los más recientes como el del Dr. Hawn de las falsas clonaciones humanas. Fraudes hay en la historia de la ciencia a cargo de personajes tan prestigiosos como Darwin, Méndel y Newton quienes, como muestra Freeland (2006), manipularon u ocultaron datos para hacer más fuertes sus teorías.

Otra problemática que entra en la gestión de la investigación es todo lo que vincula la calidad de la investigación. La ISO ha promulgado normas en este sentido tales como la serie UNE: 166000-1 de terminología en I+D+I la 166000-2 de gestión de la I+D+I gestión terminología y evaluación de proyectos. BSI 7001- 1 Guide for I+D+I Management.

Para la distinción y los ejemplos de fraude ver: Freeland, Horace. Anatomía del fraude científico. Barcelona: Drakontos, 2004 y sobre la necesidad de aumentar la revisión en la ciencia, ver cfr. y Küng, Hans. Ciencia y ética mundial. Barcelona: Trotta, 2005. 
Estas normas tienen como referente la gestión de la investigación ya sea en cuanto a proyectos o en cuanto a centros de investigación y aplican el ciclo PHVA característico de los sistemas de gestión de la calidad. El ciclo de la gestión integral se considera el eje categorial central de la gestión de la investigación.

A lo anterior debe incorporarse la problemática de la calidad de la investigación en relación con la evaluación de los sistemas de investigación: producción e investigación sobre indicadores, mejoramiento de aplicaciones computarizadas para el seguimiento de la investigación con referencia a proyectos, líneas de investigación, organizaciones, sistemas y cooperación internacional. Más adelante se presenta el concepto fundamental de la gestión de la investigación desde el cual se generan todo el campo de estudio.

\section{De la investigación y la gestión a la ges- tión de la investigación}

Los temas que dan como resultado el numeral anterior han sido parte de las preocupaciones de los autores de la Research Management Review (RMR): "The Journal of the National Council of University Research Administrators" (NCURA) ${ }^{7}$ que desde 1959 trabaja sobre: el avance en el campo del conocimiento de la administración de la investigación con programas de educación, desarrollo profesional y de la experiencia que fomentan una comunidad profesional colegial y respetada.

La revista RMR realiza revisiones de temas relacionados con la gerencia de la investigación. Es una amplia gama de problemáticas que afectan la administración de la investigación en los niveles nacional e internacional. La RMR "es un foro para la discusión del conocimiento sobre el estudio y la práctica de la profesión de la administración de la investigación. Parte de los artículos de la edición más reciente (Vol. $15 \mathrm{~N}^{\circ} 2$ de 2007) incluye artículos sobre: El alcance de la responsabilidad social en el entorno de la in- vestigación de la universidad; el comportamiento del investigador que conduce al éxito en la financiación y las universidades y los problemas de patentes. Dichos artículos son analizados más adelante.

De esta manera se muestra que el reduccionismo de la gestión de la investigación al manejo de dineros y recursos de investigación e investigadores en una institución frecuente, pero a este momento, insostenible. Se debe reiterar el énfasis en que se gestionan sistemas de investigación que tienen fuertes implicaciones en el desarrollo de los países en el marco de las sociedades del conocimiento (el plural hace referencia a un doble sentido tanto en la existencia de diversas sociedades tales y en la divergencia de concepciones de tales sociedades), así como en el mejoramiento de la calidad de vida de las poblaciones, si tenemos en cuenta una discusión tan relevante como contemporánea sobre la pertinencia y la responsabilidad de la investigación realizada desde la institucionalidad de las sociedades modernas.

Este reduccionismo tiene una especificidad en la Educación Superior. La OECD ("Organisation for Economic Co-operation and Development") en su vínculo dedicado a la gestión en educación "Managenment Education", presenta el conjunto de publicaciones y de estudios sobre gestión de la investigación en este campo:

Los gobiernos han estado dando un énfasis sin precedentes a la investigación como motor clave para el desarrollo de la sociedad del conocimiento y la nueva economía. No es de sorprender que las instituciones de educación superior estén enfocando mayores esfuerzos hacia la investigación, el prestigio en este campo, su capacidad para llevarla a cabo y el financiamiento requerido para apoyarla. Es aún menos sorpresivo ver que las políticas de investigación y la gestión de la investigación están enfrentando retos severos.

Ver en www.ncura.com. 
En primer lugar se muestra claramente la diferencia entre gestión de la investigación y gestión del conocimiento desde una perspectiva conceptual que se profundiza más adelante.

La publicación ${ }^{8}$ se plantea como una ayuda para "entender diversas perspectivas en torno a la multiplicidad y complejidad de aspectos que las instituciones de educación superior tienen que enfrentar en respuesta a la creciente presión para "producir" investigación que les mantenga en un nivel competitivo." Aquí se muestra la necesidad de una parte el campo de gestión de la investigación específico en la Educación Superior. De otra parte la vinculación entre investigación en el contexto de la Educación Superior su relación con el contexto socio económico de los países desde la perspectiva competitiva, que no es la única pero que para el objetivo central de esta digresión es lo que se quiere enfatizar.

Ahora bien la misma publicación muestra que "Utilizando ocho estudios de caso, este reporte se enfoca en los siguientes cuatro temas centrales: 1. La creciente importancia de la misión de investigación en la educación superior. 2. El fortalecimiento de las estructuras y procesos de gestión de la investigación. 3. Financiamiento y manejo de recursos de la investigación universitaria. 4. Estrategias para estimular la formación de investigadores.

Los estudios de caso incluyen instituciones en Australia, Bélgica, Brasil, Alemania, Irlanda, Malasia, Portugal y Turquía. Esta publicación será de interés para líderes institucionales de la educación superior, oficinas de investigación, formuladores de políticas, estudiantes de posgrado, investigadores y cualquier persona u organización interesados en la gestión de la investigación. Esto muestra el interés de investigar en el campo de la gestión de la investigación por que de lo contrario ¿Qué son estos estudios de caso? No es del caso mostrar en este numeral las conclusiones de los estudios. Sin embargo sí vale apuntar la distancia que hay entre la gestión de la investigación en contextos de países más innovadores y cómo en cuestión no se puede reducir a mencionar el porcentaje de la inversión en relación con el $\mathrm{PIB}^{9}$ que no muestra en nuestro país un panorama muy halagüeño, pero que no debe paralizar la acción investigativa. Por el contrario, debe servir para buscar alternativas de gestión inteligentes, creativas y sinérgicas.

Otro reduccionismo frecuente es olvidar la historia de la ciencia y las lecciones que para la gestión de la investigación se pueden aprender. Es reduccionista también por que desde una mirada la perspectiva de la gestión a la historia de la investigación o a la historia de la ciencia que no es lo mismo, se puede comprender mejor que han existido diferentes formas de producción investigativa, de las cuales la actual es otra y resultante de un proceso complejo que estamos por entender en su amplitud y profundidad. Dichos procesos se vinculan con la dinámica económica, política y social de los contextos regionales, nacionales e internacionales. No cabe duda que la investigación de grupos sociales o de fenómenos locales tiene un significado diferente en esta década que hacia mediados del siglo pasado y esto por no hacer mayores contrastes.

En este punto específico la historia de la (Investigación Acción Participativa) IAP resulta ser muy ilustrativa. La IAP se desarrolla a partir de los años setenta en nuestro país y responde a necesidades propias. Si contrastamos con sus inicios, ya no se encuentra en discusión su validez epistemológica. Se discute sobre su aporte. Tampoco está en discusión si existe un método único de la IAP. Se comprenden una serie de estrategias de investigación que no necesariamente forman parte

\footnotetext{
$8 \quad$ University Research Management: Meeting the Institutional Challenge" (Gestión de la Investigación en las Universidades: Respondiendo a los retos institucionales), 2004. Revista de la OECD. Publicaciones de IMHE.

9 Según los datos del observatorio de ciencia y tecnología la cifra apenas pasa del $0.5 \%$.
} 
de su constitución. Existe una relatividad de lo que se considera IAP en relación con sus fines que ya no son estrictamente políticos como lo fueran en principio y lo cual generara una persecución política a sus investigadores. Hoy forma parte con mayor o menor rigor, del acervo investigativo en la academia de las diferentes ciencias sociales.

Es un proceso de gestión en la medida en que se desarrolla una nueva actitud frente al trabajo de campo y los resultados no sólo son en el orden de lo cognitivo sino también involucran lo político y la trasformación de poblaciones y entornos culturales.

No es casual por ejemplo, que una perspectiva investigativa como la IAP naciera en nuestro contexto colombiano y generara en su momento tanta resistencia, no sólo política sino académica y que hoy sin más reflexión sea reconocida como una de las tendencias vitales para el desarrollo de las ciencias sociales y de la posibilidad de aplicación de tecnologías e investigación aplicada. Hoy sabemos en parte gracias a ello que la participación de las comunidades es fundamental para el desarrollo y que el conocimiento y el saber popular es válido y representa una alternativa al estancamiento descriptivista de las ciencias sociales.

Por último, también es reduccionista el olvido histórico, por que los campos de investigación a los cuales se refiere son amplios y disímiles. No sólo se realiza investigación en el contexto académico y los nuevos modos de producción de conocimiento señalados por varios autores (Gibbons, 2003; Arocena y Suzt, 2000) ${ }^{10}$ Solo rastrear las formas de cambio en la producción de conocimiento en el planeta y en Latinoamérica, hace evidente la necesidad de estudiar de manera diacrónica la gestión de la investigación y los cambios surgidos y todo lo que podemos aprender de esta reflexión conciente, de esta autopoiésis.

No es una ocurrencia para este artículo que forma parte de un estado del arte más amplio. Ya en La- tinoamérica Rodrigo Arocena y Judith Sutz (2000) señalan que:

The conditions of knowledge production are changing everywhere. The evidence of change is manifold: it includes the steady stagnation of the public budget of universities, the rising of the 'call for projects' modality as a main source of support, the growing importance of research contracts with industry and the 'agonising' processes of evaluation of research proposals (Ziman, 1994). These evidences are not geographically concentrated (Senker, 1999): it is easy to find important similarities in the recent literature on these issues regardless of their origin, be it Europe, the United States or Latin America.

Estos contextos investigativos entrecruzados señalan una dirección muy importante entorno a la gestión de la investigación latinoamericana que, subsidiaria y mantenida en el supuesto rigor de doctores en diversas áreas formados en el exterior (que varios casos no vuelven a investigar después de su formación de doctores o que nunca vuelven al país o que nos iluminan desde el exterior) implican un intercambio de las producciones provenientes de esfuerzos investigativos éticos, pertinentes que solo pueden surgir de iniciativas de investigadores residentes.

La posibilidad de generar desarrollo o de la pertinencia y significatividad de la investigación en el contexto nacional depende no sólo de aumentar la inversión sino de generar la capacidad para comprender y formular problemas propios. Lo anterior no significa hacer investigación solo para un contexto. Significa que las repercusiones y la generalización de los resultados de la investigación dependen en gran medida de formas muy complejas de la disciplina de la cual de hable. No es lo mismo esto en la física que en la economía o en la psicología que en campos emergentes como el de los estudios en propiedad intelectual en el derecho

10 El artículo de Arocena y Sutz (2000) muestra los cambios en la producción de conocimiento para el caso más específico de América Latina. 
o los sistemas integrales de calidad en ingeniería y administración. Ni sus procesos ni sus resultados proceden de la misma forma ni representan el mismo enfoque.

Las condiciones señaladas son de capital importancia para la gestión de la investigación, por que señalan que la investigación actual sucede o se produce en un contrato y obedece a una demanda. No sucede necesariamente por la lógica del encadenamiento de proyectos basada en la resolución de problemas, ni en la lógica de la falsación [Pooper] y tampoco en la del mantenimiento de núcleos fuertes [Lakatos]; esto para referirnos a la discusión que en el marco de la filosofía de la ciencia mantienen los dos autores legendarios mencionados.

Por ahora basta señalar que el objetivo de relatar algunos de los reduccionismos más notorios ha sido cubierto, lo cual abre el panorama de la gestión de la investigación como un campo disciplinar, conceptual, necesario y prometedor para el desarrollo nacional.

La pregunta epistemológica por la lógica o no del cambio en la investigación es básicamente una pregunta de mega gestión de la investigación. Esta pregunta y su discusión ocuparon gran parte de la producción de la filosofía anglosajona de la ciencia y generó un núcleo de polémica especial entre Sir Karl Popper y Thomas Samuel Kuhn"1 , pero por ahora basta con abrir la discusión. Su desarrollo sugiere una investigación que se encuentra en curso y de otra parte excede las posibilidades de presentación de este artículo.

\section{Relaciones y diferencias de la gestión de la investigación}

Dado que en nuestra cultura el campo de la gestión de la investigación puede ser relativamente reciente, es necesario establecer algunas precisiones que más que demarcar, buscan vincular y relacionar la gestión de la investigación con categorías enfoques y tendencias actuales, en relación con la producción de conocimiento.

Básicamente se realiza una discusión en relación con la gestión de ciencia y tecnología y el aparatoso uso que tiene esta denominación con respecto a la producción actual de conocimiento, incluso en relación con el derecho de la propiedad intelectual. En segundo lugar, se discute sobre la gestión del conocimiento que proviene de las ciencias administrativas y tiene también una perspectiva más restringida referente a la gestión de la investigación.

Como se verá, uno de los resultados de las discusiones es la justificación de referirnos a un campo como de gestión de la investigación muy relacionado con las entidades anteriormente señaladas, pero necesarias de diferenciar. En otras palabras, esta organización categorial responde a la pregunta ¿Por qué hablar de gestión de la investigación como un campo intelectual y no sólo de gestión?

\section{Gestión de ciencia y tecnología: relacio- nes y diferencias con la gestión de la investigación}

La gestión de ciencia y tecnología proviene de una tradición ligada a la concepción científica del Círculo de Viena de la ciencia unificada. Su énfasis entonces ha estado centrado en las denominadas "ciencias duras", y su relación de pertinencia en el momento actual se fundamenta en la recepción que tenga el sector productivo de los resultados de investigación. Se trata de utilizar el conocimiento en el entorno de aplicación para el desarrollo. Es la tendencia central en la actualidad del diseño de políticas en los gobiernos, sobre todo de los llamados países en vías de desarrollo y su forma particular de análisis de la realidad es procedimental, propositiva y monocausal.

Procedimental por que se trata de acciones con respecto a logros. Está situada en la sociedad proce-

$11 \quad$ Para una profundización de la discusión Popper Kuhn, ver Enciclopedia Iberoamericana de filosofía. Volumen IV. “La ciencia: estructura y desarrollo". Madrid: Editorial Trotta, 1993 y Mantilla, William. La recepción de la filosofía analítica en Colombia. (tesis de maestría meritoria), 2000. 
dimental y estratégica de las acciones, medios, fines que Habermas tomara críticamente de Weber. En este mismo sentido la actividad científica es heterónoma y los indicadores son externos a los procesos mismos de la investigación desde el punto de vista cognitivo. Se basan en $\%$ de investigadores, $\%$ de doctores, centros y proyectos de investigación, nivel de la inversión, indicadores de publicaciones. Los indicadores para Colombia son bastante deficientes.

Para no redundar en ello, se puede considerar para el caso específico en educación superior el "Informe sobre la educación superior en iberoamérica 2006" del proyecto CINDA/UNIVERSIA coordinado por José J. Bunner. Para el caso colombiano los datos del Observatorio Colombiano de Ciencia y Tecnología, que involucran los siguientes aspectos que sirven en parte, para ratificar los indicadores antes mencionados: gasto en ciencia y tecnología, personal en ciencia y tecnología, educación superior. En todos estos indicadores como se puede verificar en la página respectiva, el desempeño colombiano es bajo, incluso con respecto a varios de los países latinoamericanos.

Los indicadores de ciencia tecnología e innovación están vinculados con los procesos de medición de las actividades de generación, uso y difusión del conocimiento científico, el desarrollo tecnológico y la innovación organizacional permiten tener un conocimiento más cabal de estos ámbitos y mejorar la toma de decisiones de los mismos.

Propositiva por que su discurso se basa en propuestas y recomendaciones a los gobiernos para el fomento de la actividad científica y tecnológica. Incluso las condiciones en las cuales esto es viable y la asesoría correspondiente que se puede dar. Estas recomendaciones son generales para regiones de países. Se basan en estudios externos contratados desde organismos internacionales, con sus propias metodologías de recolección de datos. Los estudios son estadísticos y formulan recomendaciones generalmente de política de ciencia y tecnología coherentes con los indicadores que manejan.
Desde esta perspectiva la política de ciencia y tecnología ha sido entendida como un proceso de administración de sistemas nacionales para estimular, desarrollar y la actividad científica y su relación con la empresa. El informe de las Naciones Unidas, "que se debe citar como: Proyecto Milenio, Naciones Unidas, 2005. Innovación aplicación de los conocimientos al desarrollo". Que tiene una publicación realizada por Colciencias presenta en resumen las siguientes recomendaciones:

- Es necesario aumentar la inversión en educación científica tecnológica, y (sic) debe cambiar el papel que desempeñan las universidades

- Es necesario que los gobiernos fomentan (sic) las actividades empresariales en ciencia, tecnología e innovación

- Estimular la creación y expansión de la pequeña y mediana empresa

- Incubadoras de empresa

- Zonas de procesamiento de exportaciones

- Liberar el capital financiero

- Utilizar las adquisiciones oficiales para estimular el desarrollo tecnológico

- Aumentar la participación en el comercio internacional

- Proteger los derechos de los inventores fomentando al mismo tiempo el desarrollo tecnológico de los países en desarrollo

- Atraer la inversión extranjera directa y convertirse en parte de las cadenas de valor globales

Promoción de mercados regionales

- Establecer una estructura de asesoría

- Fortalecer la capacidad de las academias cientí- 
ficas y técnicas para participar en actividades de asesoría

- Capacitar a los encargados de tomar las decisiones de ciencia y tecnología e innovación

- Utilizar técnicas de prospectiva para fijar prioridades de financiación y formulación de las políticas

- Fortalecer la capacidad de las Naciones Unidas para utilizar en sus actividades la asesoría científica y tecnológica

- Examinar el impacto de las organizaciones encargadas de la formulación de reglas y de fijación de normas en la capacidad de los países en desarrollo para usar la tecnología y fomentar el desarrollo

- Incrementar la capacidad de las instituciones multilaterales y bilaterales para fomentar la innovación tecnológica

- Ampliar el acceso ilimitado a las publicaciones científicas

- Mejorar los beneficios y los riesgos de tecnologías nuevas existentes.

Las veinte recomendaciones componen en desglose, la totalidad del informe. Combinan la relación entre producción científica tecnológica innovación y producción económica.

Lo que se asume en este informe y en la postura general adoptada por la gestión de ciencia y tecnología, es que el conocimiento es la fuerza más importante para producir riqueza y generar desarrollo sostenible con equidad para la población: "Si bien la innovación tecnológica no es la única fuente de transformación económica, su importancia aumentará con el tiempo. Aunque el informe rechaza la opinión en el sentido de que la tecnología determina el cambio socioeconómico, sí toma atenta nota de la evolución conjunta entre el cambio técnico y el ajuste social" (Ídem: 41). Es claro que supera la indicación de informes anteriores que se fundamentan en un modelo monocausal en la relación tecnología - desarrollo.

A esto hay que agregar las diversas formas que confluyen con ciertos cambios en las formas de mostrar innovación- desarrollo; conocimiento científico - desarrollo entre otras. Es la manifestación de un planteamiento de ventaja competitiva basado en el conocimiento disponible. La Universidad como institución social y política del conocimiento, se encuentra como elemento central de la relación con el sector productivo que significa de manera preponderante su relación con las PYMES: "Se ha reconfigurado un nuevo modelo productivo, pasándose de un modelo basado en la producción y economías de escala, a otro que se basa en la distribución y economías de amplitud según los requerimientos de un mercado con exigencias crecientes"..."La nueva ventaja competitiva de una región es el conocimiento disponible en la misma, las formas en las que se facilite el intercambio de conocimiento, capacitación y aprendizaje regional, cadenas productivas financiamiento disponible, infraestructura y telecomunicaciones" (ídem). Todo un conjunto de aspectos, de los cuales la relación conocimiento productividad constituyen el eje central.

Los casos exitosos desde esta perspectiva son evidentes y contundentes. Incluye países tan disímiles culturalmente como Estados Unidos y Corea, en momentos históricos igualmente diferenciables. Son además los ejemplos a seguir según los expertos nacionales y la discursividad o recursividad más plausible, es por lo menos, iniciar el camino que eleve nuestros indicadores en este sentido con el énfasis en la inversión y la búsqueda de estrategias de fomento y estímulo en el sector empresarial.

No es gratuito entonces que las "agendas de gestión de ciencia tecnología y desarrollo" inicien con el punto dedicado a la financiación de investigación y las comparaciones, que saltan a la vista explican en el sentido causal del término la poca competencia y capacidad de 
un país como Colombia o sus afines para integrarse a la sociedad del conocimiento y aprovechar sus beneficios vinculados al cambio socioeconómico.

Las agendas regionales en el nivel de Colombia, muestran la reproducción que se hace de este modelo de gestión de ciencia y tecnología. Además de verificar este punto en el plan estratégico actual de Colciencias, es claro también en la agenda regional de ciencia, tecnología e innovación del Departamento de Bolívar.

El objetivo central dice:"Promover la cultura de C,T e I en las regiones como camino hacia la sociedad del conocimiento, buscando hacer los territorios más competitivas (sic) en la economía globalizada del conocimiento, como nuevo marco de referencia,...". Si se observa este objetivo desde el análisis semántico, su planteamiento muestra que la necesidad del conocimiento desde la perspectiva de la gestión de ciencia y tecnología no está claro en relación con las metas de reducción de la pobreza o la forma más optimista como es planteada en la generación de riqueza, a partir de la producción de conocimiento.

Desde un análisis pragmático queda reducido a un círculo vicioso pues es producir conocimiento para este mismo fin. Esto pone en evidencia el mecanicismo del cual se ha hecho advertencia anteriormente.

Desde la perspectiva epistemológica y los avances desde la sociología del conocimiento, la denominación de "ciencia y tecnología" no toma en cuenta la pluralidad metodológica y la diversidad de disciplinas. Todo ello proveniente de las repercusiones del programa fuerte de la sociología ${ }^{12}$. Desconocer esta producción de la sociología del conocimiento implica dejará atrás los avances metodológicos y epistemológicos de las ciencias sociales y su importancia como conocimiento generador de desarrollo.
Por esto no sería adecuado ni preciso utilizarla para denominar el campo interdisciplinar. Esto obliga a continuar con la idea de gestión de la investigación. Hasta ahora se ha hecho una distinción con el campo disciplinar de las ciencias de la administración o de la administración académica. Así, como aquí apenas se ha mencionado la necesidad de la discusión epistemológica de la gestión de la investigación en el sentido de observar las teorías, por ejemplo, de la filosofía analítica desde la perspectiva constructiva de la gestión de la investigación como categoría, no de establecer estatuto de la gestión de la investigación como disciplina o menos ciencia, también debemos plantear la necesidad de una discusión de los aportes del relativismo científico y sus consecuencias en la gestión de la investigación. Todos ellos problemas sin resolver, pero que hacen pensar en un campo de conocimiento, en una dinámica de compresión necesaria de los procesos para mejorar las oportunidades de generar políticas de cambios en nuestros países latinoamericanos.

\section{Apéndice de artículos aplicados}

Además de la bibliografía señalada y los artículos de NCURA básicos para esta investigación, gran parte de los datos señalados proceden de la interpretación de un conjunto de artículos como objetos aplicados de gestión de la investigación, varios campos muy actuales como el de la gerencia informática, la gestión de ordenadores, la gestión de tecnología, la gestión de la información climatológica, los sistemas de gestión e información sobre conocimiento etc, que muestran unas tendencias y de los cuales se ha realizado una interpretación cualitativa que compone este escrito. A continuación se muestran sus referencias en Internet y sus datos básicos. A manera de conclusión algunos de los aportes que han sido incorporados al artículo.

12 Para profundizar en el programa fuerte y sus efectos y análisis más actuales ver: Beltrán Villalva, Manuel. Perspectivas sociales y conocimiento. México: Anthropos - Unam, 2000 Ibáñez, Jesús. Nuevos avances en la investigación social. Barcelona: Anthropos, 1998. 


\section{Relación de documentos electrónicos de aplicación de gestión del conocimiento y de la investigación en diferentes áreas alternativas}

1 Ciencia, tecnología, sociedad y valores. Educar para participar en la sociedad del conocimiento. Curso de formación docente por Internet. Edición para el Cono Sur. España. Organización de Estados Americanos para la Ciencia y la Cultura.

2 Ciencia, Tecnología, Sociedad e Innovación en Ibero América Cátedra CTS+I - Perú Universidad Nacional de Ingeniería responsables del módulo: José Luis Solleiro (UNAMI) y Luis Javier Jaramillo (OEI) http://www.oei.es/catedraperu

3 La Universidad Latinoamericana del Futuro Tendencias - Escenarios - Alternativas. Uruguay Editada por la Organización de Estados Iberoamericanos para la Educación, la Ciencia y la Cultura. Rodrigo Arocena y Judith Sutz: http://www.oei. es/revistactsi/index.html.

4 Ciencia tecnología y sociedad. España, Universidad de Oviedo, Marcelo Valerio y Walter Antonio Bazzo http://www.oei.es/bolctsi.htm.

5 “Plan valenciano de investigación científica, desarrollo tecnológico e innovación". España Consejería de Innovación y Competitividad Subsecretaría de la Oficina de Ciencia y Tecnología. http://www. gva.es/industria/idi/castellano/pdf/gestion.pdf

6 “Ciencia, Tecnología e Innovación en los países menos desarrollados España", Revista Madrid+d, Raquel Andino. http://www.madrimasd.org/revista/revista24/aula/aula2.asp

7 Algunos resultados de la evaluación efectuada por la CNEAI de la actividad investigadora realizada por profesores Universitarios y personal científico del CSCl en 2002 y 2003. España, Revista Madrid+d Marcelo Valèrio http://www.madrimasd.org/revista/revista24/aula/aula2.asp
8 Ciencia, tecnología y sociedad del conocimiento: nuestro futuro. España. http://www.prouinvestigaciomilitar.org/documents/eleccions2004/ RmesDPSOE.pdf

9 Sistema Español de Ciencia y Tecnología. España Ministerio de Educación y Ciencia. Ministerio de Educación y Ciencia http://www.mec.es/ciencia/ cte/files/Sistema-IDI.pdf

10 Consejo Nacional de Ciencia y Tecnología. Perú RED ScienTI. "Red internacional de Fuentes de Información" http://www.concytec.gob.pe/scienti/scienti@concytec.gob.pe

11 Conclusiones y recomendaciones. grupo de trabajo ciencia y salud. Brasil Cuarta reunión regional de coordinación de la biblioteca virtual en salud (bvs): grupo bvs/ciencia y salud. Grupo de trabajo Ciencia y Salud. http://bvs4.icml9. org/public/documents/recommendations/BVSCyS-Conclusiones-2005.pdf

12 Gestión de Sistemas de Investigación Universitaria en América latina Venezuela Instituto Universitario de Tecnología José Antonio Anzoátegui. Jaim Royero http://www.rieoei.org/deloslectores/ 412Royero.pdf

13 I Seminario Nacional de Gestión Tecnológica e Innovación: Argen-TEC I. Argentina I Seminario Nacional de Gestión Tecnológica e Innovación: Argen-TEC I. Asociación latinoamericana de gestión Tecnológica (altec), http://www.altec.secyt. gov.ar/pdfs/seminario_argen_tec_l.pdf

14 La gestión centralizada de los ordenadores de los centros tic's de la consejería de educación de la junta de Andalucía España El centro de gestión avanzado de centros TIC. Rafael García Rivas, David Erosa García http://www.csi.map. es/csi/tecnimap/tecnimap_2006/03T_PDF/centr o\%20de\%20gestion\%20avanzado.pdf 
15 Análisis de un año de Malware en las aapp. España Centro de gestión avanzado (c.g.a.):

Rafael García Rivas Director del C.G.A. y jefe del Departamento de Informática Educativa en la D.G. de inovación Educativa y Forrmación del Profesorado. David Erosa García Programador del C.G.A. de la D.G. de Innovación Educativa y Formación del Profesorado. http://www.csi. map.es/csi/tecnimap/tecnimap_2006/03T_PDF/ centro\%20de\%20gestion\%20avanzado.pdf

16 Cevalsi: el observatorio valenciano para la sociedad tecnológica y del conocimiento España CEVALSI Elena Llorca Asensi DNI: 21488066. ellorca@ovsi.com. Fundación OVSI (Oficina Valenciana para la Sociedad de la Información). Elena Ruiz González Fundación OVSI (Oficina Valenciana para la Sociedad de la Información) Verónica Morte Egea. http://uww.csi.map.es/csi/ tecnimap/tecnimap_2006/03T_PDF/cevalsi\%20el \%20observatorio\%20valenciano.pdf

17 Complementando a métrica: proceso de ingeniería de requisitos de seguridad para el desarrollo de sistemas de información seguros España SI (Seguridad de Ingeniería Métrica) Daniel MeIlado Fernández Técnico Superior de Informática Gerencia de Informática de la Seguridad Social. Eduardo Fernández-Medina Patón. Universidad de Castilla-La Mancha. Mario Piattini Velthuis. Universidad de Castilla-La Mancha. http://www.csi. map.es/csi/tecnimap/tecnimap_2006/03T_PDF/ complementando\%20a\%20metrica.pdf

18 Centros tic aplicados a la gestión. séneca y pasen España Centros DIG, TIC, Software libre, SENECA, PASEN. María A. Barceló Martínez. CEIP "Menéndez y Pelayo". http://uww.csi.map.es/csi/ tecnimap/tecnimap_2006/03T_PDF/centros\%20ti c\%20aplicados\%20a\%20la\%20gestion.pdf

19 Computación y criptografía cuánticas: retos para la seguridad en la sociedad de la información España Centros DIG, TIC, Software libre, SENECA, PASEN.
María A. Barceló Martínez http://www.csi. map.es/csi/tecnimap/tecnimap_2006/03T_PDF/ centros\%20tic\%20aplicados\%20a\%20la\%20gesti on.pdf

20 Del libro al dato España. El INE Armando de la Torre del Río. Instituto Nacional de Estadísticahttp://www.csi.map.es/csi/tecnimap/tecnimap_ 2006/03T_PDF/centros\%20tic\%20aplicados\%20a \%20la\%20gestion.pdf

21 El" clima", una herramienta para la gestión de la información climatológica en Andalucía España Subsistema de Información de Climatología Ambiental (CLIMA). José Manuel Moreira Madueño. Mariano Corzo Toscano. Ma Fernanda Pita López. Carmen Guerrero de Mier. http://www.csi.map. es/csi/tecnimap/tecnimap_2006/03T_PDF/el\%2 0clima\%20una\%20herramienta\%20para\%20la\% 20gestion.pdf

22 Investigaciones sobre Ciencia, tecnología y Desarrollo Humano en Cuba Cuba Publicada con el patrocinio de las naciones Unidas para el desarrollo (PNUD) Editada por Mercie Group Enpses Cujae, La Habana Dirigida por el centro de Investigaciones de la Economía Mundial (CIEM) http://www.ciem.cu/proyectos/proy/8_Investigac

23 Herramientas para la innovación regional: el impulso desde la administración a las PYMES España Ministerio de Administraciones publica Sevilla Juan José Goñi Zabala Instituto Ibermática de Innovación (Grupo Ibermática http://www.csi.map. es/csi/tecnimap/tecnimap_2006/tema_03.htm

24 Computación y criptografía cuánticas: retos para la seguridad en la sociedad de la información España Ministerio de Administraciones publica Sevilla Alberto Villafranca Ramosa Ministerio de la Presidencia http://www.csi.map.es/csi/tecnimap/tecnimap_ 
25 Gestión integral del centro de proceso de datos de la seguridad social España Ministerio de Administraciones publica Roberto Valtueña Rincón. Gerencia Informática de la Seguridad Social

La mayoría de los artículos presentados datan de investigaciones sobre ciencia y tecnología, diseño de herramientas estadísticas, la generación de centros TIC aplicados a la gestión, computación y criptografía: retos para la seguridad en la sociedad de la información, desarrollo de sistemas de información cada vez más seguros, complementaciones métricas a los procesos de ingeniería, entre otros.

La información allí presentada muestra de forma sintética que los intereses se centran en aspectos tales como:

- Investigar, realizar un seguimiento de las innovaciones y desarrollo normativo de las tecnologías de la información y comunicación, así como de establecer la relación con otras administraciones públicas

- Servicios concentrados a la sociedad en la administración de servidores y redes locales de la Red de Centros TIC, Centro de Atención a Usuarios, y de soporte de conectividad al resto de los centros educativos públicos de niveles no universitarios de Andalucía, así como el mantenimiento y actualizaciones de paquetería del Sistema Operativo (SO).

- Potenciar los elementos más competitivos del Sistema y a la resolución de problemas o deficiencias del mismo.

- Avanzar decididamente en la convergencia europea en estas materias, para propiciar que nuestro país esté en condiciones de construir un brillante futuro.

- Tales avances científicos aplican herramientas metodológicas precisas en campos disciplinares específicos, son experiencias que sin duda alguna han colocado a los países en niveles más altos de desarrollo y competencia internacional en el contexto globalizado actual.

Dentro de la producción intelectual señalada en los artículos el mayor porcentaje de la producción intelectual se concentra en el diseño cada vez más preciso y sofisticado de herramientas de aplicación.

Cabe resaltar sin embargo, el artículo que se presenta sobre Jaim Royero titulado "Gestión de sistemas de investigación universitaria en América Latina" el cual propone una " comprensión sobre la relación histórica entre el sistema científico-tecnológico y la sociedad en su conjunto, dado que la actividad científica y tecnológica es concebida como estrategia social para la superación de la pobreza y del atraso social, para Tûnnerman(2002) "el subdesarrollo científico tecnológico es, a la vez causa y consecuencia del subdesarrollo económico-social" (pág. 172).

Sin embargo, salvo algunas de las excepciones planteadas en general, los artículos presentados denotan que dentro del contexto de las ciencias aplicadas básicas se puede leer de manera implícita una articulación con los procesos de gestión de la investigación, debido a que dentro de su estructura se establece la producción cada vez más avanzada de tecnología, considerando esta la razón más prioritaria, para el desarrollo de la sociedad.

La gestión de la investigación es un proceso externo y basado en resultados. Es reconocida como una gestión externa al proceso de producción de conocimiento la relación investigación - desarrollo. Esto lleva a la postura básica de los primeros momentos de la gestión del conocimiento, que se basa en la integración de los resultados de conocimiento al desarrollo y la intención de que la producción de conocimiento esté fundamentada en ese aspecto dentro del marco de una organización empresarial.

De esta manera se evalúa la pertinencia y la significatividad del conocimiento en relación con su aplicación al desarrollo económico. 
Sin embargo, dada la tendencia política de los países latinoamericanos, genera una preocupación por mostrar resultados de manera que se base en estadísticas externas a los procesos de producción de conocimiento.

\section{Bibliografía}

Beltrán Villalva, Manuel (2000). Perspectivas sociales y conocimiento. México: Anthropos - Unam.

Bunner, José J. (coordinador para el caso colombiano. Datos del Observatorio Colombiano de Ciencia y Tecnología) "Informe sobre la educación superior en Iberoamérica 2006" Proyecto CINDA/UNIVERSIA,

Deming Calidad, Productividad y Copetitividad: la salida de la crisis. Madrid: Diaz Santos, 1989.

Enciclopedia Iberoamericana de Filosofía. Volumen IV. "La ciencia: estructura y desarrollo". Madrid: Editorial Trotta, 1993.

Freeland, Horace (2004). Anatomía del fraude científico. Barcelona: Drakontos.

Guibons Michel (1997). Los nuevos modos de producción del conocimiento. Barcelona: Pomares.

Ibáñez, Jesus (1998). Nuevos avances en la investigación social. Barcelona: Anthropos.

Juan Carrión Maroto, Introducción conceptual a la gestión del conocimiento. En www.gestión del conocimiento.com, 2007

Küng, Hans (2005). Ciencia y ética mundial. Barcelona: Trotta.

Machado Cano, María J. Acimed 2006; 14(6) Cartas Consultoría para la gestión de la investigación y la publicación en la provincia Ciego de Ávila. En: www.acimed.cu
Mantilla, William (2000). La recepción de la filosofía analítica en Colombia. Universidad Santo Tomás. (Tesis de maestría meritoria).

(1999). "Gerencia planeación y evaluación en organizaciones educativas". Informe Colciencias.

(2000). Gerencia y educación. Conceptos y experiencias. Bogotá: Universidad Externado Colciencias.

Proyecto Milenio, Naciones Unidas, 2005. Innovación aplicación de los conocimientos al desarrollo.

Rodrigo Arocena a, Judith Sutz. Changing knowledge production and Latin American universities. En: Research Policy 30 (2001) 1221-1234. Department of Science and Development, Faculty of Sciences, Universidad de la República, Montevideo, Uruguay Academic Department, University Research Council. Received 1 November 1999; received in revised form 25 July 2000; accepted 18 October 2000

Royero, Jaime: "Gestión de sistemas de investigación"...OEI- Revista Iberoamericana de Educación.

Shewhart Economic Control of Quality Of manufactured Product. Mac Millan, Nueva York: Reeditado por la American Society For Quality Control, 1980.

Tunerman, Carlos (2000). Universidad y sociedad. Caracas: Ministerio de Educación Cultura y Deportes.

University Research Management: Meeting the Institutional Challenge", $\mathrm{N}^{\circ} 1$ 2004. Revista de la OECD. Publicaciones de IMHE. 